تاثير مستخلص السماد العضوي (X-Humate 85) وطريقة الإضافة في نمو وحاصل أثجار المشمش

\author{
فاروق فرج جمعة \\ استاذ \\ جامعة بغداد/ كلية الزراعة \\ عبد الستار جبار حسين \\ باحث علمي \\ وزارة العلوم والتكنلوجيا/ دائرة البحوث الزراعية \\ www.asjh_67@yahoo.com
}

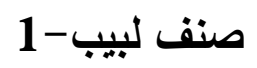

رجاء عبد الهادي كاظم

استاذ مساعد

جامعة بغداد/ كلية الزراعة

المستخلص

نفذ البحث على أثجار المشم صنف لبيب-1 بعمر 11سنة العائدة الى كلية الزراعة- جامعة بغداد/ أبو غريب خلال موسمي النمو 2013 و2014 لمعرفة تأثير مستخلص السماد العضوي وطريقة اضافته في مقاييس النمو والحاصل. اضيف المستخلص في باية الربيع بطريقتين الأولى رشاً على الأولق بالتراكيز 1 و2 و3 غم.لتر -1 والثانية إضافة أرضية بالتراكيز 2 و4 و6 غم.لتر -1 واستخدم تصميم القطاعات العشوائية الكاملة والبرنامج الجاهز Genstat في تحليل البيانات. أظهرت النتائج في الموسم الأول ان رش المستخلص على الأوراق بالتركيز العالي اعطى اعلى القيم لصفات اطوال الافرع ومساحة الورقة ونسبة العقد بلغت 12.39 سم و20.86 سم² و

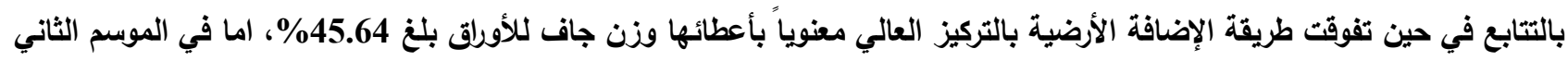
فقد كاتت النتيجة عكسية تماماً. كما سببت طريقة الرش بأعلى تركيز محتوى عالي من الكلوروفيل في الأولق وللموسمين بلغ 28.68 و31.22 وحدة SPAD بالتتابع الا ان اعلى حاصل ثمار ظهر عند إضافة المستخلص الى التربة بالمستوى نفسه ليصل 16.957 و18.207 كفم.شجرة-1 للموسمين بالتتابع. لذا فأن طريقة الاضافة الارضية بتركيز 6غم.لتر -1 كاتت الافضل عند التسميد بهذا بانه

المستخلص.

الكلمات المفتاحية: رش ورقي، تسميد عضوي، حامض الهيوميك

The Iraqi Journal of Agricultural Sciences -1108-1114: (4) 48/ 2017

Kadhim \& et al.

\title{
EFFECT OF ORGANIC FERTILIZER EXTRACT ( X - HUMATE 85 ) AND APPLICATION METHOD ON GROWTH AND YIELD OF APRICOT TREES
}

\section{LABEEB-1 CV.}

R. A. Kadhim

University of Baghdad

College of Agriculture
AS. J. Hussein

Ministry of Science and Technology

Agricultural Research office

www.asjh_67@yahoo.com

\section{ABSTRACT}

This rsearch was conducted on apricot trees cv. Labeeb at the college of Agriculture - University of Baghdad/ Abu Gharib during the growing seasons 2013 and 2014, to investigate the effect of organic fertilizer extract and the method of application on growth and yield parameters, the extract was applied in the biggning of spring by two ways the first foliar application with three concentrations 1,2 and 3 g. $\mathrm{L}^{-1}$ and second ground application 2,4 and 6 g.L $\mathrm{L}^{-1}$. The experiment were designed using Randomized Complete Block Design (R.C.B.D) and Genstat program for statistical analysis. Results of the first season showed that the foliar spraying with high concentration gave highest values for branches length, leaf area and fruit set percentage $(12.39 \mathrm{~cm}),\left(20.86 \mathrm{~cm}^{2}\right)$ and $(10.62 \%)$ respectively, while the ground application superior in the high level by gaving leaves dry weight was $45.64 \%$, but the results completely differed in the second season. The foliar spraying with higher concentration caused high content of chlorophyll in the leaves during both seasons (28.68) and (31.22) SPAD unit respectively, while applying of extract in the soil with high level showed highest yield quantity (16.957 and $18.207{\mathrm{~kg} . t r e \mathrm{e}^{-1}}^{\text {) }}$ respectively for both seasons. Ground application was the best method for fertilization with $\mathrm{X}$-Humate85 extract.

Key world: foliar application, organic fertilization, humic acid 
الهيوميك سواء كانت رشاً على الأوراق او إضافة أرضية

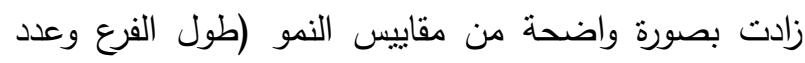
الأوراق والمساحة الورقية والحاصل والصفات الفيزيائية والكيميائية للثمار منل صلابة الثمار و وSSC للعصير ونسبة ولهن

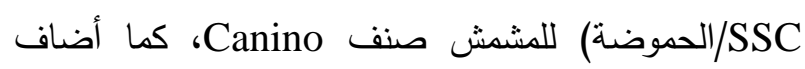
Shaddad

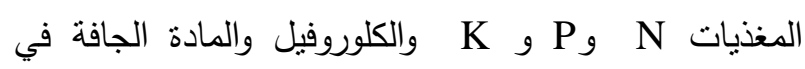
الأوراق. لذا فالهذف من هذا البحث هو تحسين نمو اشجار المشمش صنف لبيب وزيادة كمية حاصل الثمار ومعرفة تأثير طريقة اضافة مستخلص السمادالعضوي.

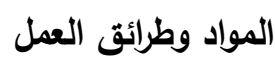
أجريت الدراسة في بستان المشمش التابع الى كلية الزراعة/

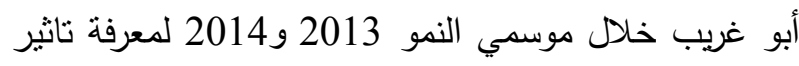
المستخلص العضوي 85 X-Humate في نمو وحاصل أشجار المشمش صنف لبيب بعمر 11 سنة.===|ضيف المستخلص (جدول 1) في بداية الربيع بطريقتين احداهما

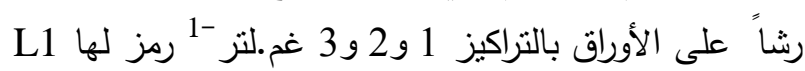

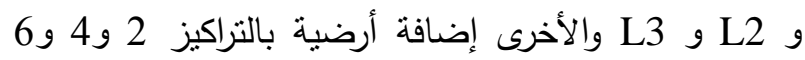

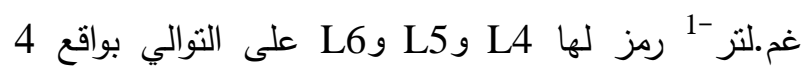

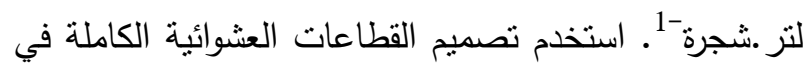
تجربة حقلية تضمنت 6 معاملات بثلاثة مكررات لكل معاملة وعدة الثجرة كوحدة تجريبية وحللت البيانات احصائيات

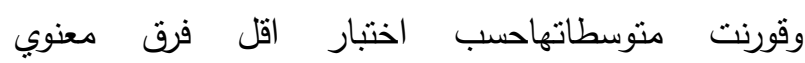
Least Significant Difference (L.S.D) احتمال 5\% واستعمل البرنامج الجاهز Genstat في التحليل

الاحصائي (20).
المشم.Prunus armeniaca L. هو احد أنواع الفاكهة ذات النواة الحجرية التي تعود الى العائلة الوردية Rosaceae واثمارها ويمكن ان تبدء بالاثمار بعد 3-4 سنوات والحمل

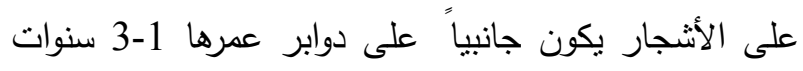
(8). تعود أهمية ثمار المشمش الغذائية الى محتواها المرتفع دائي من فيتامين A و النياسين niacin مقارنة بثمار الفواكه

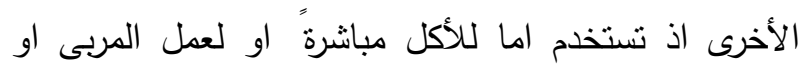
العصير كما يمكن ان تجفف وتعلب وتنتخدم نواة ثمار المشمش الحلوة في صنع الحلويات وفي استخلاص الزيت من البذور المرة (2). ان استخدام المنتجات الطبيعية في

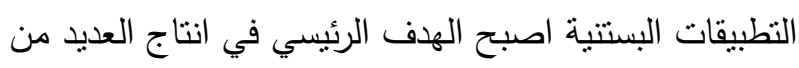
أنواع الفاكهة، اذ تحسن الأسمدة العضوية الصفات الفيزيائية

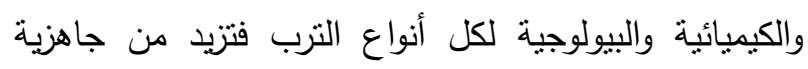
العناصر الغذائية وتعادل حموضة التربة ومن هذه الأسمدة

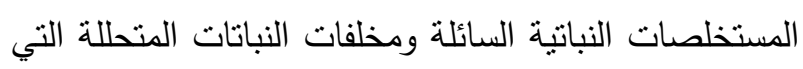
يمكن ان تؤثز في ظروف المنطقة الجذرية والمجموع الخضري فضلا عن ان انتاج هذه المخلفات بعملية التخمر يتم من خلالها استخلاص الاحياء الدقيقة ومن ثم ثمن نموها ومضاعفتها كالبكتريا والفطريات وغيرها (13) وزيادة فعالية

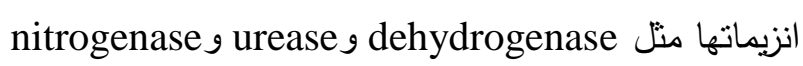
(3)، كما تحسن الأسمدة العضوية سلوك العديد من العناصر في الترب من خلال مجاميعها الفعالة Fulvic و مumic acid التي لها القدرة على احتجاز العناصر في صيغ معقدة

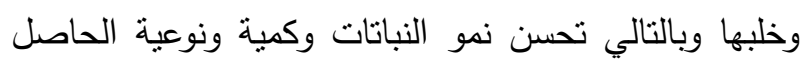

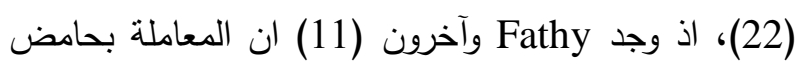

جدول 1. يوضح مكونات المستخلص العضوي 85 Xوئشرات الدراسة

\begin{tabular}{|c|c|c|c|}
\hline القيمة & الصفة & القيمة & الصفة \\
\hline $\begin{array}{l}\% 3.0-0.6 \\
\% 10 \\
\% 5.0-1.0 \\
11-9\end{array}$ & $\begin{array}{c}\mathbf{N} \\
\mathbf{K}_{2} \mathbf{0} \\
\mathbf{P}_{2} \mathbf{0}_{5} \\
\mathbf{P H}\end{array}$ & $\begin{array}{l}\% 65 \\
\% 15 \\
\% 85 \\
\% 85\end{array}$ & $\begin{array}{r}\text { Humic acid } \\
\text { Fulvic acid } \\
\text { الذقاوبانة }\end{array}$ \\
\hline
\end{tabular}

2. مساحة الورقة (سم²): استخدم جهاز قياس المساحة 1. اطوال الافرع ومحيط الساق (سم): استخدم شريط القياس الورقية Leaf Area Meter نوع CI-20 امريكي الصنع لهنه

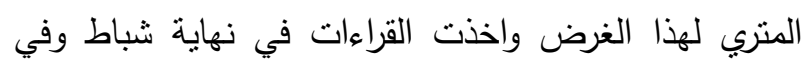
الذي يعمل بطريقة المسح الضوئي. 
في الموسم الثاني اذ تفوقت طريقة الاضافة الارضية

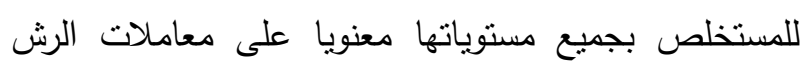
والمقارنة ولاسيما التركيز العالي L6 الذي اعطى زيادة بلغت معند

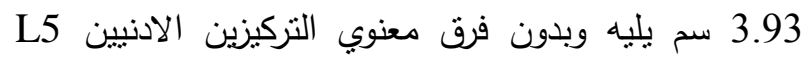

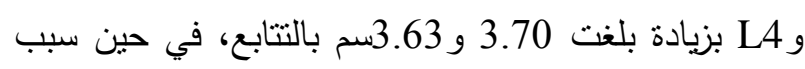
رش المستخلص بأعلى تركيز L3 انخفاض في معدل الزيادة في محيط الساق الى ادنى حد وللموسمين وكانت القيم 1.73

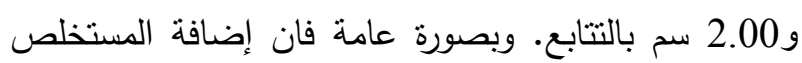

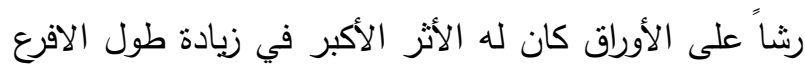

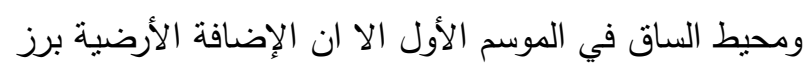
تأثنرها في الموسم الثاني. قد يعزى سبب الزيادة في اطوال

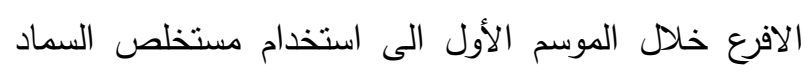

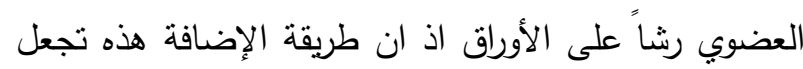

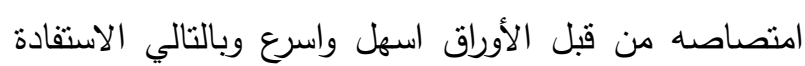
المباشرة منه كونه يحتوي على المغذيات الضرورية للنمو ولا سيما البوتاسيوم الذي يعمل على تحفيز الانزيمات الخاصة بالعمليات الفسلجية كالبناء الضوئي وكذلك يساعد في حركة وانتقال الغذاء المصنع في الأوراق الى انسجة النبات الأخرى

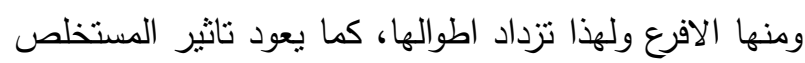
عند إضافته الى التربة في الموسم الثاني الى عامل الوقت اللازم لتغلغله في التزبة وتأثثره في صنفاتها الفيزيائية والكيميائية اذ يعمل على خفظ PH التربة وزيادة جاهزية المغذيات الموجودة أساساً في التربة لامتصاصها من قبل فيل

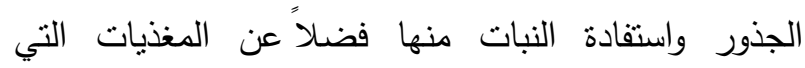
يضيفها المستخلص نفسه الى التربة وهنا يكون التأثير

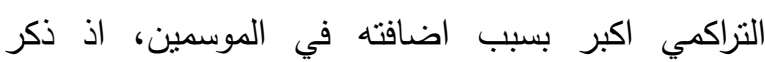
Eman دوراً مهماً في تحسين خواص التربة الفيزيائية والكيميائية والبيولوجية كما انه مستودع للعديد من العناصر الغذائية

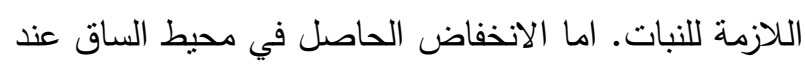

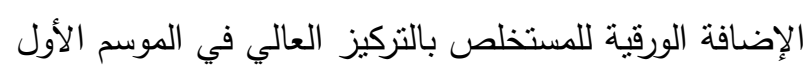
فربما يعود الى تتجيعه النمو الخضري العلوي للنبات (جدول

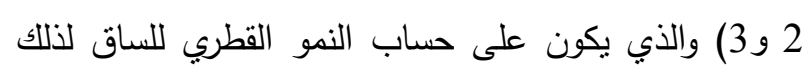

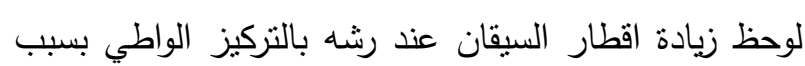

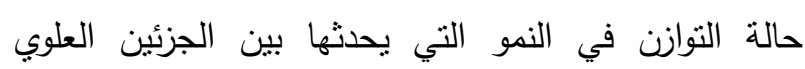

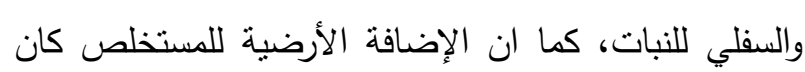

3. الكلوروفيل في الأوراق (SPAD): قيس حقلياً جهاز

.SPAD

4. المادة الجافة في الأولق (\%) (كP): وزنت 20 ورقة منتخبة قبل التجفيف ثم جفقت في فرن كهربائي على درجة حرارة 65 م" لحين ثبوت الوزن ثم وزنت مرة أخرى بميزان حساس واستخرجت نسبة المادة الجافة بموجب المعادلة:

$100 \times$

المادة الجافة (\%)

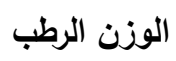

5. نسبة العقد (1\%): حسبت على ثلاثة افرع منتخبة اذ جرى الرى حساب عدد الازهار الكلي في مرحلة الازهار الكامل ثم حسبت عدد الثمار العاقدة (بعد العقد) وبموجب المعادلة:

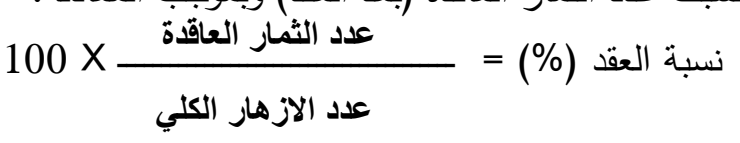
6. انتاجية الثمار (كغم.شجرة-1): استخرجت بضرب عدد الثمار الكلي X معدل وزن الثمرة.

النتائج والمناقشة 1- الزيادة في اطوال الافرع ومحيط الساق: بينت النتائج في الجدول 2 ان المستخلص العضوي اثز معنوياً في اطوال الافرع لاشجار المشمش ولكلا الموسمين ولكن هذا التاثير كان متفاوت بين طريقتي الإضافة ، ففي الموسم الأول اعطى الرش بالتركيز L3 اعلى زيادة

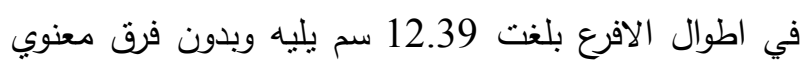

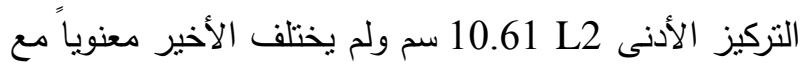
الإضافة الأرضية للمستخلص بالتركيز العالي L6 الذي نتج

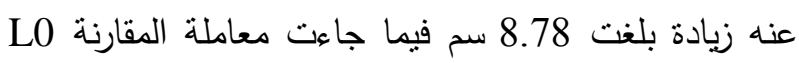
بأقل زيادة لهذه الصفة وكانت 3.91 سم. اما في الموسم

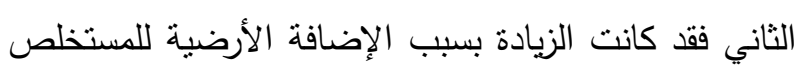
بالتركيز العالي L6 اذ بلغت 14.10 سم تلتها وبفارق معنوي إضافة التركيز L5 بزيادة 10.79 سم وهذه بدورها تقوقت معنوياً على معاملة الرش الورقي بالتركيزين L3 و L23 التئي

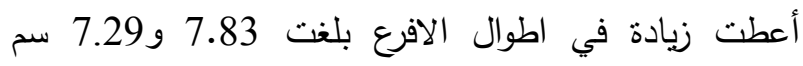
بالتتابع قياساً بمعاملة المقارنة L0 التي نتج الأنج عنها اقل زيادة

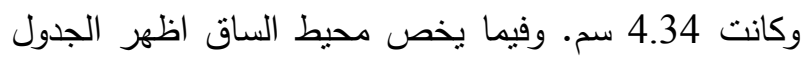

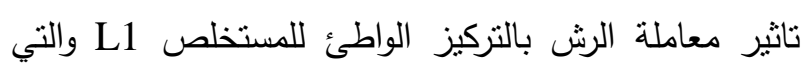

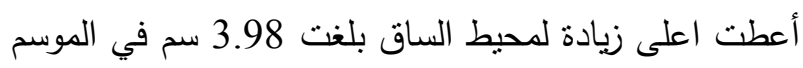

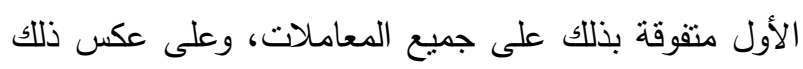


وأتنقت الننائج مع ما وجده Al-Hawezy (6) من ان

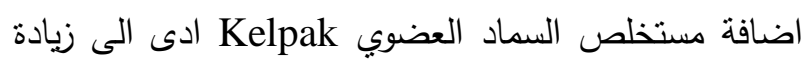
قطر الساق الرئيس لاشجار البشملة.
لها تأثيرها في الموسم الثاني لربما بسبب قرب السيقان من

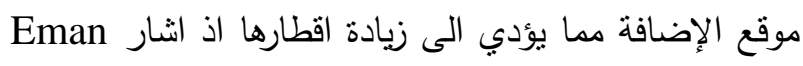

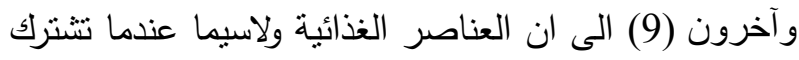
مع حامض الهيوميك حسنت النمو الخضري الثتلات الزينتون

جدول 2. تأثير مستخلص السماد X-Humate 85 وطريقة اضافته في اطوال الافرع ومحيط الساق (سم).

\begin{tabular}{|c|c|c|c|c|c|c|}
\hline \multirow{2}{*}{\multicolumn{2}{|c|}{ الزيادة في محيط الساق (سم) }} & \multirow{2}{*}{\multicolumn{2}{|c|}{ مالزيادة في اطوال الأفرع (سم) }} & \multirow{2}{*}{\multicolumn{2}{|c|}{ فرت تلتريز }} & \multirow[t]{2}{*}{ المعامة } \\
\hline & & & & & & \\
\hline 2.50 & 2.59 & 4.34 & 3.91 & Lo & 0 & المقارنة \\
\hline 2.83 & 3.98 & 5.94 & 7.87 & L1 & 1 & \\
\hline 2.56 & $\begin{array}{l}3.00 \\
1.73\end{array}$ & 7.29 & $\begin{array}{l}10.61 \\
12.39\end{array}$ & L2 & 2 & ر رش ورقي \\
\hline 3.63 & 2.50 & 6.50 & 5.43 & L4 & 2 & \\
\hline 3.70 & 2.86 & 10.79 & 6.67 & L5 & 4 & أضافة أرضستسة \\
\hline $\begin{array}{l}3.93 \\
0.58\end{array}$ & $\begin{array}{l}2.46 \\
0.91\end{array}$ & 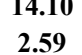 & $\begin{array}{l}8.18 \\
2.12\end{array}$ & & & \\
\hline
\end{tabular}

الثاني وذلك بتميز طريقة التسميد الأرضي على سواها. ان

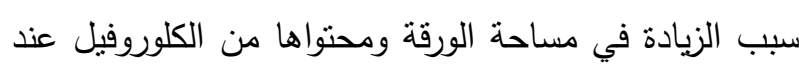

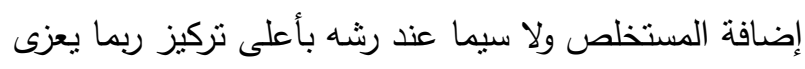

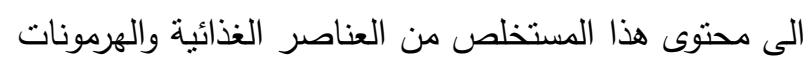

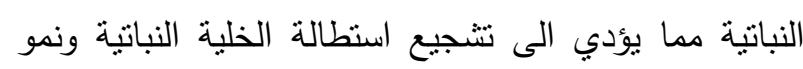

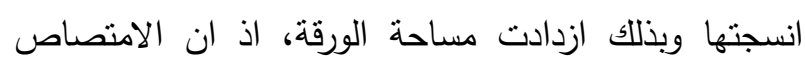

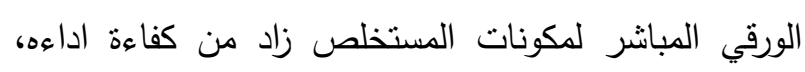

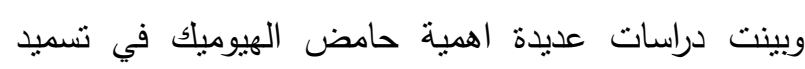

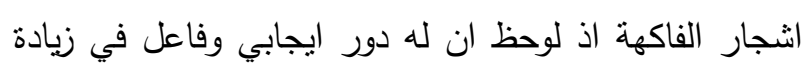

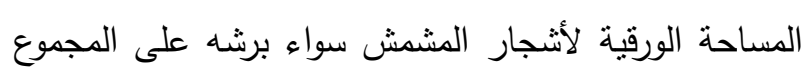

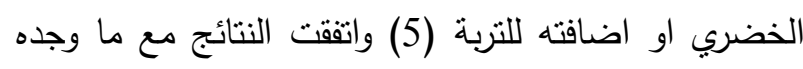

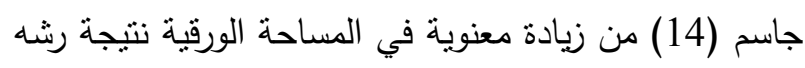

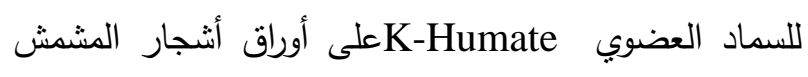

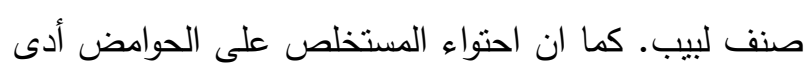

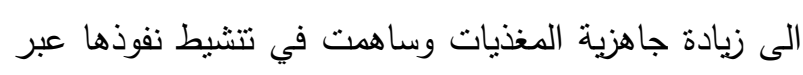

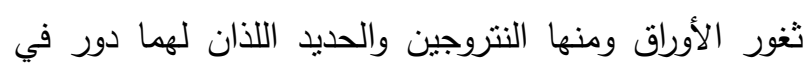
K- بناء الكلوروفيل، اذ وجدت Omnia ( humate تعمل على زيادة اخذ عنصري الحديد والمنغنيز

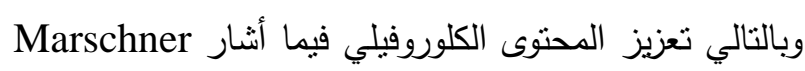

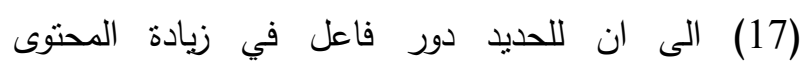

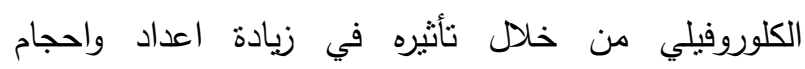

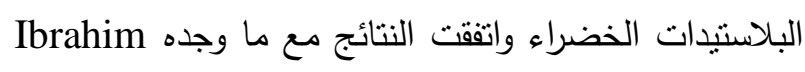
وآخرون (12) بأن محتوى الأوراق من الكلوروفيل ارتفع وانداث
2. مساحة الورقة ومحتوى الأورلق من الكلوروفيل

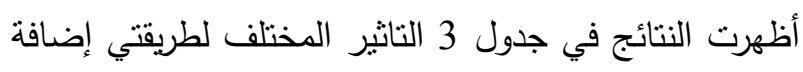

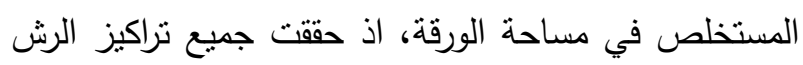
تفوقاً معنوياً في الموسم الأول وذلك باعطائها اكبر الأوراق

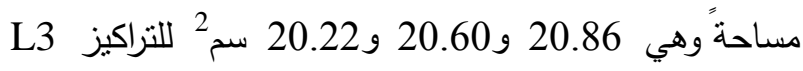

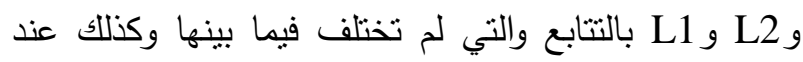

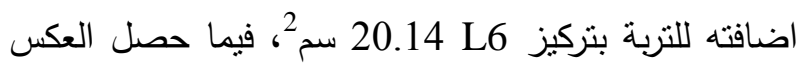

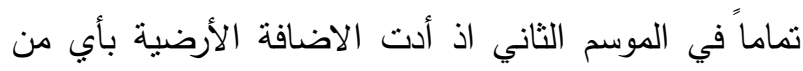

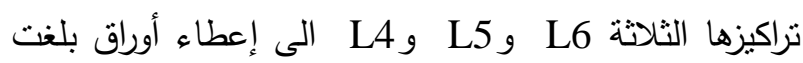

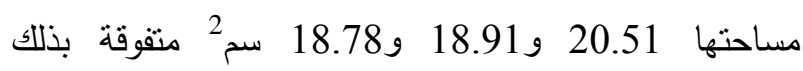
معنوياً على المعاملات الأخرى ومن ضمنها معاملة المقارنة

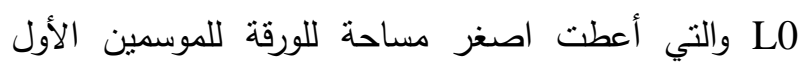

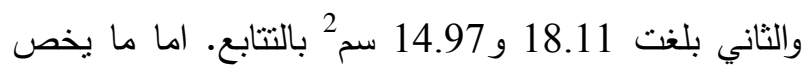
محتوى الأوراق من الكلوروفيل فقد كان لمعاملة الرش الثان التاني بالثركيز العالي L3 الحظ الاوفر وذلك بإعطائها اكبر محتوى لكان

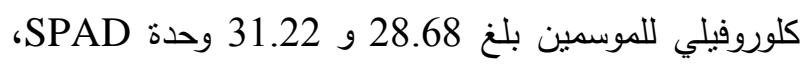
L2 تلتها وبدون فرق معنوي معاملة الرش بالتركيز الثاني وكان 28.14 وحدة SPAD في الموسم الأول والاضافة الأرضية بالتركيز العالي الموسم الثاني فيما ظهر اقل محتوى للكلوروفيل في الأوراق

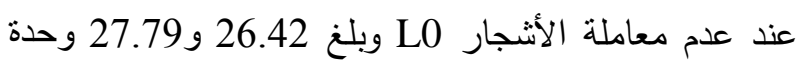
SPAD

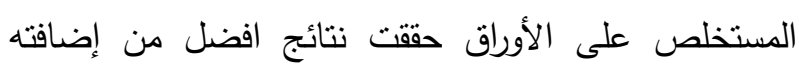

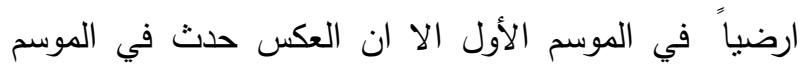




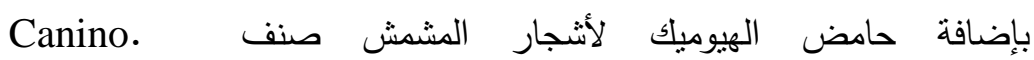

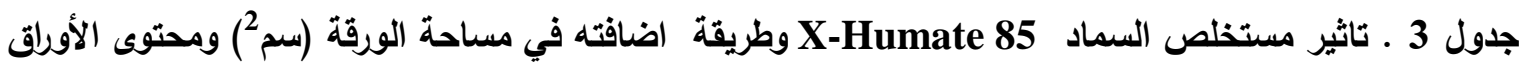
(الكلوروفيل (وحدة SPAD)

\begin{tabular}{|c|c|c|c|c|c|c|}
\hline \multicolumn{2}{|c|}{ الكلوروفيل (وحدة SPAD) } & \multicolumn{2}{|c|}{ مساحة الورقة (سم²) } & \multicolumn{2}{|c|}{ التركيز غم.لتر -1 } & \multirow[t]{2}{*}{ المعاملة } \\
\hline 2014 & 2013 & 2014 & 2013 & & & \\
\hline 27.79 & 26.42 & 14.97 & 18.11 & LO & $\mathbf{0}$ & المقارنة \\
\hline 28.52 & 27.32 & 15.99 & 20.22 & L1 & 1 & \\
\hline 28.55 & 28.14 & 17.74 & 20.60 & L2 & 2 & رش ورقي \\
\hline 31.22 & 28.68 & 17.61 & 20.86 & L3 & 3 & \\
\hline 29.43 & 26.99 & 18.78 & 19.53 & L4 & 2 & \\
\hline 30.44 & 27.26 & 18.91 & 19.89 & L5 & 4 & أضافة أرضية \\
\hline 30.71 & 27.29 & 20.51 & 20.14 & L6 & 6 & \\
\hline 0.77 & 0.57 & 1.14 & 0.87 & & & \\
\hline
\end{tabular}

ربما تعود الى ما بحتويه هذا المستخلص من احماض

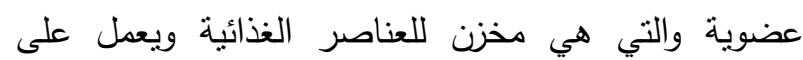
تحسين التبادل الكاتيوني وزيادة نسبة المغذيات الجاهزة في التربة مما يسهل من امتصاصها من قبل الجذور وزيادة

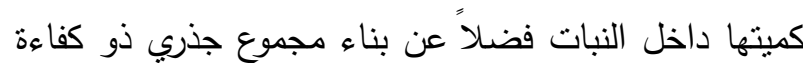
عالية في امتصاص المغذيات المختلفة مما يشجع من عملية البناء الضوئي وزيادة نراكم المواد المصنعة في الأوراق

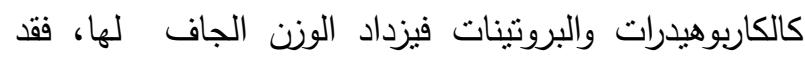

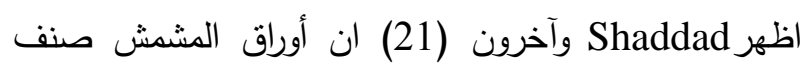
Canino حامض الهيوميك الى التربة كما ان هذا المحتوى هن الكاربوهيدرات والبروتينات والمغذيات الكبرى والصغرى ربما يكون له تأثير إيجابي في زيادة عقد الثمار وابقائها بدون

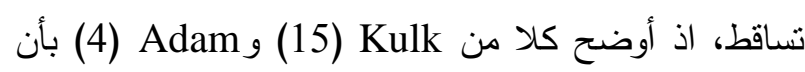
التحسن في نسبة عقد الثمار يظهر نتيجة لزيادة انبات حبوب ونات اللقاح.

4. إنتاجية الثمار (كفم.شجرة-1) أوضحت النتائج في الجدول 5 ان الإضافة الأرضية لأي من

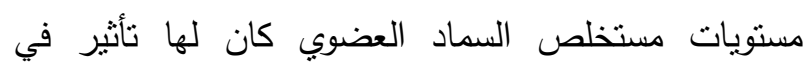
إنتاجية شجرة المشمش وللموسمين، اذ تفوقت طريقة الإضافة هذه معنوياً على باقي المعاملات ولا سيما إضافة التركيز العالي L6 الذي اعطى اعلى حاصل للثمار بلغ 16.957

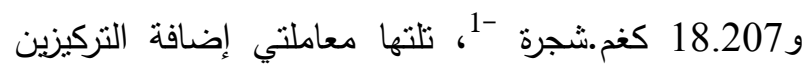
الادنيين L5 و L4 بإنتاجية بلغت 14.247 و 15.833

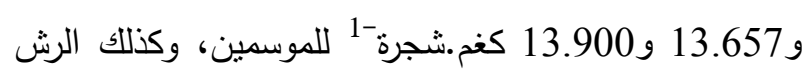

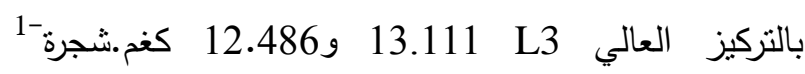

3. نسبة المادة الجافة وعقد الثمار (\%)

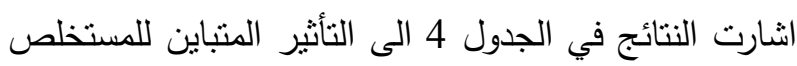

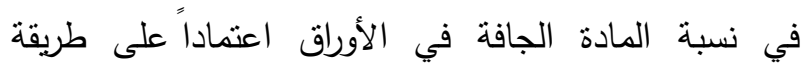
الإضافة فكان للإضافة الأرضية بالتركيز العالي L6 الأثر الأرئ

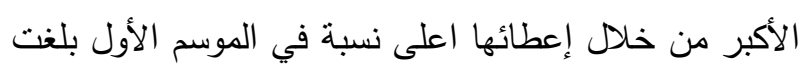

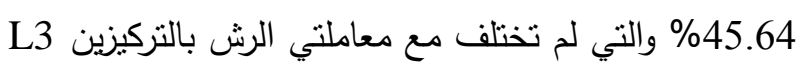

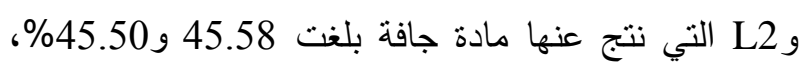

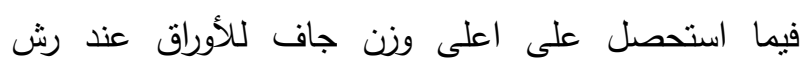
المستخلص بأعلى تركيز L3 في الموسم الثاني فكانت فكانت نسبته 46.04\%، اما اقل نسبة للمادة الجافة فقد ظهرت عند

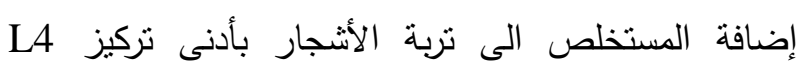
وللموسمين اذ وصلت هذه النسبة في الأوراق الى 44.38 و44.17\% بالتتابع. ومن ناحية أخرى فقد اثرت المعاملة

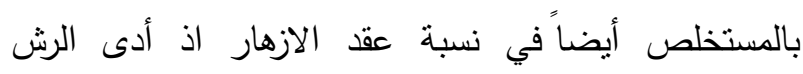

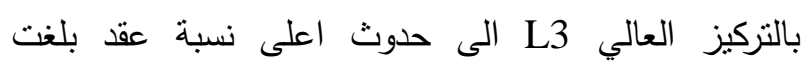

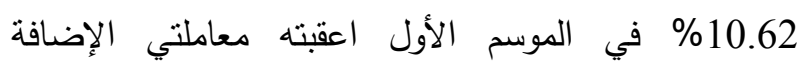

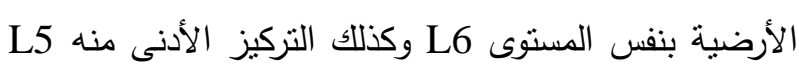

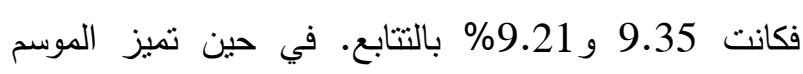
الثاني بأكبر عدد للثمار العاقدة عند إضافة المستخلص الى بلى في

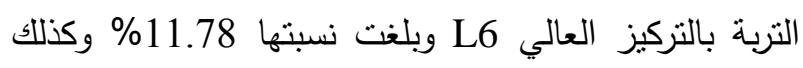

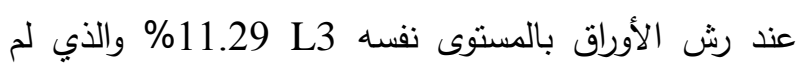

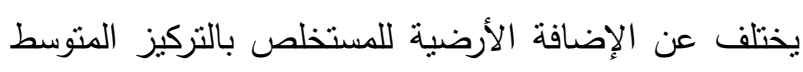
L5 اذ كانت نسبة العقد فيه 11.10\%، فيما أعطت الأشجار الأرضية غير المعاملة L0 اقل عدد للثمار العاقدة بلغت نسبتها 3.57 التها و5.26\% للموسمين بالتتابع. ان زيادة الوزن الجاف للأوراق

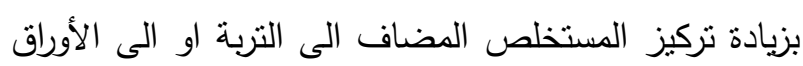


الكاربوني وتراكم المواد المصنعة بهذه العملية وانتقالها من الأوراق والافرع الى الثمار وبالتالي زيادة عقد الثمار وعددها ومعدل وزن الثمرة وبالمحصلة النهائية ازدادت إنتاجية الثمار ، وأثنار Mansour وآخرون (16) الى تفوق معاملة العناصر المغذية مع حامض الستريك في حاصل اشجار الكمثرى صنف Lecont، واتفقت النتائج مع ما وجدته Omnia) (19) عند إضافة محلول K-humate على نباتات الكرز اذ حصلت على احسن انتاج وكانت نسبة الزيادة في البلوبري 30 وفي الرزبري 50\%.
للموسمين بالتتابع مقارنة مع الأشجار غير العاملة L0 التي أعطت اقل إنتاجية وكانت 9.324 و 8.297 كغم.شجرة-1 بالتتابع. ان التفوق المعنوي في حاصل الثمار لأثجار المشمش وللموسمين عند تسميدها أرضياً بمستخلص السماد العضوي والذي تتاسب طردياً مع مستوى التركيز المضاف قد يعود الى تأثز صفات الحاصل بالتسميد منل نسبة العقد وعدد الثمار ووزنها وذلك بسبب ما يحتويه المستخلص من محفزات النمو كالمغذيات والمكونات العضوية والهرمونية التي تشجع العمليات الحيوية داخل النبات مما يزيد من كفاءة التمثيل

جدول 4 ـ تأثير مستخلص السماد 85 X-Humate وطريقة اضافته في نسبة المادة الجافة في الاورلق وعقد الثمار (\%).

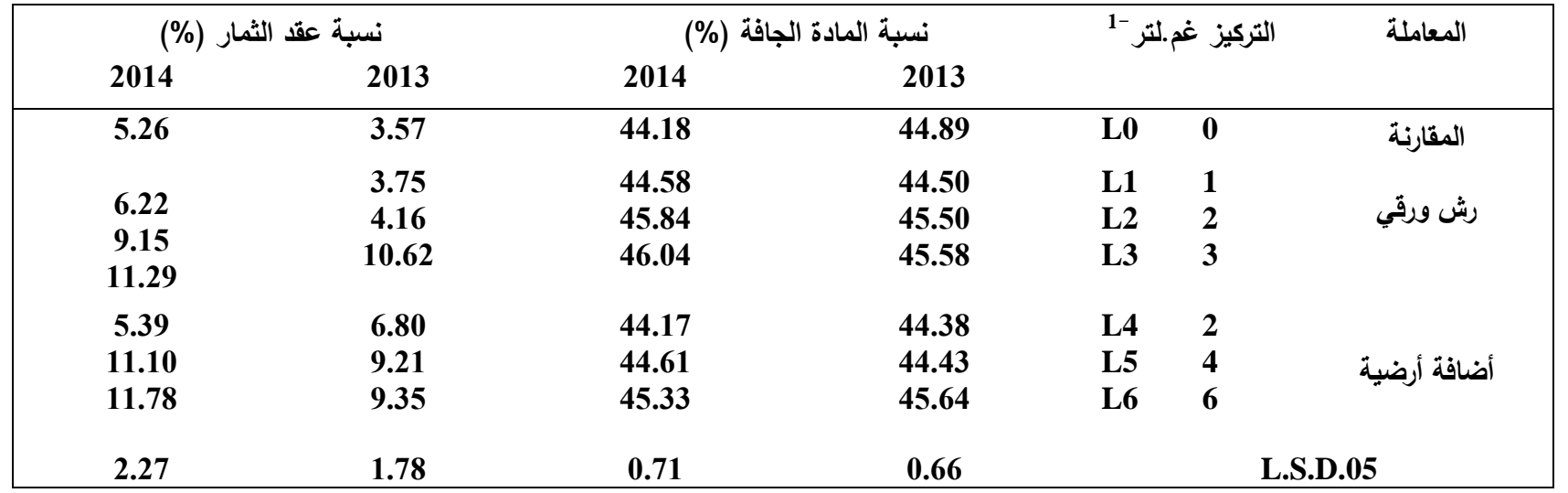

جدول 5 ـ تأثير مستخلص السماد X-Humate 85 وطريقة اضافته في إنتاجية الثمار (كفم.شجرة"1)

\begin{tabular}{|c|c|c|c|c|}
\hline \multicolumn{2}{|c|}{ إنتاجية الثمار كفم.شجرة-1" } & \multicolumn{2}{|c|}{ التركيز غم.لتر-1 } & \multirow[t]{2}{*}{ المعاملة } \\
\hline 2014 & 2013 & & & \\
\hline 8.297 & 9.324 & LO & $\mathbf{0}$ & المقارنة \\
\hline 8.967 & 9.700 & L1 & 1 & \\
\hline 9.931 & 10.162 & L2 & 2 & 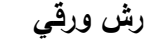 \\
\hline 12.486 & 13.111 & $\mathbf{L 3}$ & 3 & \\
\hline 13.900 & 13.657 & L4 & 2 & \\
\hline 15.833 & 14.247 & L5 & 4 & أضافة أرضية \\
\hline 18.207 & 16.957 & L6 & 6 & \\
\hline 2.263 & 2.021 & & & \\
\hline
\end{tabular}

\section{REFERENCES}

1. Association officials of analytical chemists (A. O. A. C). 1970. Official methods of analysis. $11^{\text {th }}$ ed. Washington D.C. pp.1015.

2. Apricot Farming Information Guide. India (A.F.G.). 2015.

3. Abou Hussein, S.D., I. El-Oksha, T. ElShorbagy and A. M. Gomaa. 2002. Effect of cattle manure, bio fertilizers and reducing mineral fertilizer on nutrient content and yield of potato plant. Egypt. j. Hort, 29 (1): 99- 115. 4. Adam, M. S. 1999. The promotive effect of cyanobacterium (Nostoc muscorum) on the growth of some crop plants. Acta Microbiologic polonica 48 (2): $163-171$.
5. Al-Alaf, A. H. I. 2012. The Important of Using Humic Acid as a Substitute for Miniral Fertilizer in Fruit Trees Fertilization. Extention Bulletin, College of Agriculture and ForestryUniversity of Mosul, Department of Agricultural Extension and Technology Transfer: 1-3.

6. Al-Hawezy, SH. M. N. 2014. The use of kelpak to seedlings Loquat (Eriobotya jappanica L.) International journal of Scientific and Research Publication, 4(5) :7681, ISSN 2250-3153.

7. Bortiri, E., S. -H. Oh, J. Jiang, S. Baggett, A. Graner, C. Weeks, M. Buckingham, D. Potter, D.E. Parfitt. 2001. Phylogeny and 
systematics of prunus (Rosaceae) as determined by sequence analysis of ITS and the chloroplast trnL-trnF spacer DNA. Systematic Botany 26 (4) : 797-807.

8. El-Issa, E. and M. Batha. 2012. Deciduous Fruit Production. First Edition, Damascus University Publications. Syria:181-191.

9. Eman, A. A, Abd El-Monem, S. M. ElAshry and E. A. M. Mostafa. 2011. Performance of coratina olive seedling as affected by spraying humic acid and some micro elements. Journal of Applied Sciences Research, 7(11): 1467-1471.

10. Eman, A. A., M., Abd El-Moneim, S. Saleh and E. A. M. Mostafa. 2008. Minimizing the quantity of mineral nitrogen fertilizers on grapevine by using humic acid. organic and bio fertilizers. Res. J. of Argic. and Biological Sci. Egypt. 4 (1): 46-50.

11. Fathy, M. A. , M. A. Gabr and S. A. El Shall. 2010. Effect of Humic Acid Treatments on "Canino" Apricot Growth, Yield and Fruit Quality. New York Sci. J, 3 (12): 109- 115. 12. Ibrahim, H. K, G. S. Abd El-Atif and A. A. Khalil. 2005. Effect of soil application of different treatments on growth, fruiting parameters, fruit properties and leaf nutrients content of canino apricot trees. J. Argic. Sci. Mansoura Univ., 30 (3): 1617- 1629.

13. Ingham, E. 2005. The compost tea brewing manual as printing. Soil Food Web. Incorporated, Vegan 3, 31- 32.

14- Jasim, N. A. 2008. Effect of Foliar Spray K-Humate and Type of Training and Cultar on The Development of Branchs and Vegetative Adult of Apricot Trees Prunus armeniaca. Ph.D. Dissertation. College of Agriculture University of Baghdad. Iraq:40-45.

15. Kulk, M. M. 1995. The potential for using cyanobacteria (blue-green algae in the biological control of plant pathogenic and fungi. European J. of plant pathol. 101 (6): 585-599.

16. Mansour, A.E.M, F.F. Ahmed, E.A. Shaaban and A. A. Fouad. 2008. The Beneficial of using citric acid with some nutrients for improving productivity of Leconte pear trees. Research Journal of Agriculture and Biological Science, 4(3): 245247.

17. Marschner, H. 1986. Mineral Nutrition in Higher plants. Academic press Inc. London, LTD: 65-73.

18. Omnia Specialties Australia .2005. Technical Bulletin. Enhancing chlorophyll with K- humate. Omnia Specialties Australia pty Ltd. www.Australian humates.com.

19. Omnia Specialties Australia. 2006. Khumate Increases crop yield and quality.http:/www.Australianhumates.com/pdf /QSA6R052.

20. Payne, R, M. Darren, H. Simon, B. David and S. Duncan. 2007. Genstat for Windows ${ }^{\mathrm{TM}}$ $10^{\text {th }}$ Introduction Release 10 was Developed by VSN International Ltd, in Collaboration with Practising Statisticians at Rothamsted and Other Organisation in Britain, Australia and New Zealand.

21. Shaddad, G, A. Khalil and M. A. Fathi 2005 Improving growth, yield and fruit quality of "Canino" apricot by using bio, mineral and humate fertilizers. Minufiya. J .Agric. Res. 30 (1): $317-328$.

22. Stino, R. G, A. T. Mohsen, M.A. Maksoud, M. M. M. Abd El-Migeed and A.M. Gomaa, A.Y. Ibrahim. 2009. Bio- organic fertilization and its impact on apricot young trees in newly reclaimed soil. American- Eurasian journal of Agricultural and Environmental Sciences, 6: $62-69$. 